\title{
Hierarchical Bayesian Model for Simultaneous EEG Source and Forward Model Reconstruction (SOFOMORE)
}

Stahlhut, Carsten; Mørup, Morten; Winther, Ole; Hansen, Lars Kai

Published in:

IEEE International Workshop on Machine Learning for Signal Processing, 2009. MLSP 2009

Link to article, DOI:

10.1109/MLSP.2009.5306189

Publication date:

2009

Document Version

Publisher's PDF, also known as Version of record

Link back to DTU Orbit

Citation (APA):

Stahlhut, C., Mørup, M., Winther, O., \& Hansen, L. K. (2009). Hierarchical Bayesian Model for Simultaneous EEG Source and Forward Model Reconstruction (SOFOMORE). In IEEE International Workshop on Machine Learning for Signal Processing, 2009. MLSP 2009 (pp. 1-6). IEEE. https://doi.org/10.1109/MLSP.2009.5306189

\section{General rights}

Copyright and moral rights for the publications made accessible in the public portal are retained by the authors and/or other copyright owners and it is a condition of accessing publications that users recognise and abide by the legal requirements associated with these rights.

- Users may download and print one copy of any publication from the public portal for the purpose of private study or research.

- You may not further distribute the material or use it for any profit-making activity or commercial gain

- You may freely distribute the URL identifying the publication in the public portal 


\title{
HIERARCHICAL BAYESIAN MODEL FOR SIMULTANEOUS EEG SOURCE AND FORWARD MODEL RECONSTRUCTION (SOFOMORE)
}

\author{
Carsten Stahlhut, Morten Mørup, Ole Winther, Lars Kai Hansen \\ Technical University of Denmark \\ Department of Informatics and Mathematical Modelling \\ Richard Petersens Plads, DK-2800 Kgs. Lyngby, Denmark.
}

\begin{abstract}
In this paper we propose an approach to handle forward model uncertainty for EEG source reconstruction. A stochastic forward model is motivated by the many uncertain contributions that form the forward propagation model including the tissue conductivity distribution, the cortical surface, and electrode positions. We first present a hierarchical Bayesian framework for EEG source localization that jointly performs source and forward model reconstruction (SOFOMORE). Secondly, we evaluate the SOFOMORE model by comparison with source reconstruction methods that use fixed forward models. Simulated and real EEG data demonstrate that invoking a stochastic forward model leads to improved source estimates.
\end{abstract}

\section{INTRODUCTION}

Electroencephalography (EEG) measures the electrical activity that arises from neurons in the brain by an array of sensors placed on the scalp. We are interested in reconstruction of the EEG source generators, which is known as the inverse problem with a mapping from source space to sensor space that is many to one. Despite the fact that EEG source reconstruction is a severe ill-posed problem it contains highly interesting information for the functional imaging community due to its high temporal resolution in comparison with functional magnetic resonance imaging (fMRI) and positron emission tomography (PET). The relation between the measured EEG signal and the current sources within the brain can under the quasi-static approximation of Maxwell's equation be expressed as a linear combination of the sources in the so-called forward problem. Given the measured EEG signal $\mathbf{M} \in \Re^{N_{c} \times N_{t}}$ and the current sources $\mathbf{S} \in \Re^{N_{d} \times N_{t}}$, with $N_{c}, N_{d}$, and $N_{t}$ denoting the number of channels, dipoles, and time samples, respectively, the forward relation is given by, [1],

$$
\mathbf{M}=\mathbf{A} \mathbf{S}+\mathcal{E},
$$

where the noise $\mathcal{E}$ is assumed additive. The interrelationship between the sensors and the current sources is given by the lead field matrix/forward model $\mathbf{A} \in \Re^{N_{c} \times N_{d}}$ with the rows referred to as the lead fields for the sensors and the columns as the forward fields for the sources. In this contribution we assume the orientations of the dipoles at the vertices to be perpendicular to cortex. However, a more flexible orientation can be incorporated by inclusion of three columns in A per dipole corresponding to the three directional components. Different levels of complexity of the head model exist, where the spherical head model, the boundary element method (BEM), and finite element methods (FEM) are examples of increasingly complex models, [2], [3].

To obtain a unique solution when solving the inverse EEG problem additional information or constraints such as anatomical, physiological, and mathematical properties are needed, [4]-[7]. This has led to a development of a vast number of inverse methods in the literature. One approach used is to restrict the estimated current sources to a limited number of current dipoles. This can lead to an overdetermined inverse problem where the limited set of dipoles is then fitted to the data by minimizing a measure of reconstruction error such that the location, orientation, and amplitude of the dipoles can be obtained, [8]. A different class of inverse methods is the so-called distributed models also known as source imaging techniques, where a distribution of the current sources is recovered. The well-known minimum norm (MN) estimate [9] reconstructs a source distribution that minimizes the $l_{2}$-norm of the measurements and the explained EEG by a given source configuration.

Embedding prior assumptions of the sources $\mathbf{S}$ into the reconstruction problem is conveniently implemented in a Bayesian framework with the use of the prior distribution $p(\mathbf{S})$. In fact this important choice of prior primarily differentiates the different source localization methods. Given the observed data we can now use the marginal posterior distribution as representation of the unknown sources,

$$
p(\mathbf{S} \mid \mathbf{M})=\frac{p(\mathbf{M}, \mathbf{S})}{p(\mathbf{M})}=\frac{\int p(\mathbf{M} \mid \boldsymbol{\theta}) p(\boldsymbol{\theta}) \mathrm{d} \boldsymbol{\theta} \backslash \mathbf{S}}{p(\mathbf{M})}
$$

where $\boldsymbol{\theta}$ is a set of parameters for the model including $\mathbf{S}$ and $\boldsymbol{\theta} \backslash \mathbf{S}$ denotes the parameter set $\boldsymbol{\theta}$ except for $\mathbf{S}$. To obtain the 
posterior distribution for $\mathbf{S}$ we marginalize all other parameters than $\mathbf{S}$. The distribution $p(\mathbf{M})$ is the marginal likelihood also referred to as the model evidence and is given by $p(\mathbf{M})=\int p(\mathbf{M} \mid \boldsymbol{\theta}) p(\boldsymbol{\theta}) \mathrm{d} \boldsymbol{\theta}$. The model evidence is important for model comparison. Since all parameters are integrated out to obtain the model evidence, the Bayesian framework naturally favors simpler models over more complex models that a priori can model a larger range of data sets. Unfortunately, the marginal likelihood becomes intractable for most prior distributions, and thereby the posterior $p(\mathbf{S} \mid \mathbf{M})$ cannot be computed either. Thus, approximate methods e.g. Laplace approximation [10], variational Bayesian (VB) [6], [11], and markov chain monte carlo (MCMC) sampling [12] have been used.

While existing source localization methods perform the inversion under the assumption that the forward model is known, we will treat the source reconstruction problem without making this assumption about the forward model. This is motivated by the many noise processes that contribute to the forward model, including the representation of the cortical surface, the conductivity distribution, and electrode positions. When 'realistic head models' are constructed from tissue segmentation based on e.g. structural MRI, the geometry of the head model is affected by the resolution and tissue segmentation errors. The issue of modeling the forward process was recently pursued in [12], [13] from quite a different viewpoint than ours. In [13] the basic structure of the forward model is assumed known, while the skull-brain conductivity ratio is an unknown parameter which is estimated simultaneously with a few sources in a dipole fit setting. Similarly, [12] has proposed a probabilistic approach for distributed models to account for uncertainties in the skull conductivity. Here, we apply a more unconstrained approach by modeling the whole forward model as uncertain using a prior distribution for the forward model. We propose a first attempt for distributed models to perform simultaneous source and forward model reconstruction, in short the SOFOMORE model [14]. In this paper we focus on the evaluation of the performance of modeling the forward propagation model in the SOFOMORE model in relation to the MN method.

\section{METHODS}

Given the linear relation in Eq. 1 and if we assume the noise to be time independent multivariate Gaussian distributed, the likelihood for a single time point $t$ can be expressed as $p\left(\mathbf{m}_{t} \mid \mathbf{s}_{t}, \boldsymbol{\Sigma}_{\mathcal{E}}\right)=\mathcal{N}\left(\mathbf{m}_{t} \mid \mathbf{A} \mathbf{s}_{t}, \boldsymbol{\Sigma}_{\mathcal{E}}\right)$ where $\boldsymbol{\Sigma}_{\mathcal{E}}$ is the noise covariance matrix. For simplicity we assume no temporal correlation. However, this can also be integrated as outlined in [10]. In the remainder of this paper we assume $\boldsymbol{\Sigma}_{\mathcal{E}}=\beta^{-1} \mathbf{I}_{N_{c}}$. In a minimum norm setting a multivariate Gaussian prior for the sources with zero mean and covari- ance $\alpha^{-1} \mathbf{I}_{N_{d}}$ is assumed. Moreover, it is assumed that the forward propagation model $\mathbf{A}=\mathbf{A}^{(0)}$ is known. With the use of Bayes rule Eq. 2 it is seen that the posterior distribution is maximized by

$$
\begin{aligned}
\boldsymbol{\Sigma}_{\mathbf{M}} & =\left(\mathbf{A}^{(0)}\left(\alpha^{-1} \mathbf{I}_{N_{d}}\right) \mathbf{A}^{(0) T}+\beta^{-1} \mathbf{I}_{N_{c}}\right)^{-1} \\
\mathbf{S}_{\mathrm{MN}} & =\left(\alpha^{-1} \mathbf{I}_{N_{d}}\right) \mathbf{A}^{(0) T} \boldsymbol{\Sigma}_{\mathbf{M}} \mathbf{M}
\end{aligned}
$$

It is noted that since the likelihood and prior are both Gaussian distributions, the posterior and marginal likelihood will also be Gaussian distributions. The estimation of the sources, the precision parameters $\alpha$ and $\beta$ are performed using a standard expectation-maximization (EM) scheme [11].

In contrast to the $\mathrm{MN}$ formulation we here propose a hierarchical model that incorporates corrections of the forward fields simultaneously with the source estimation. As prior for the current sources we use a zero-mean multivariate Gaussian distribution with a diagonal precision matrix $\mathrm{D}$, where the diagonal elements are $\boldsymbol{\alpha}=\left\{\alpha_{i}\right\}_{i=1}^{N_{d}}, \mathbf{s}_{t} \sim$ $\mathcal{N}\left(\mathbf{0}, \mathbf{D}^{-1}\right)$. Hereby, the dipoles can have different variances corresponding to some dipoles being expected to be more active than others. The current variances are assumed to not change over the time period $t=1: N_{t}$ and are estimated by an automatic relevance determination prior (ARD) quite similar to [6]. Thus, we have $p\left(\alpha_{i} \mid \nu_{\alpha_{i}}, \zeta_{\alpha_{i}}\right)=\mathcal{G}\left(\alpha_{i} \mid\right.$ $\left.\nu_{\alpha_{i}}, \zeta_{\alpha_{i}}\right)$ where $\mathcal{G}(\cdot)$ is the Gamma distribution with skewness parameter $\nu_{\alpha_{i}}$ and inverse scale parameter $\zeta_{\alpha_{i}}$ such that the mean is given by $\nu_{\alpha_{i}} / \zeta_{\alpha_{i}}$ and the variance $\nu_{\alpha_{i}} / \zeta_{\alpha_{i}}^{2}$.

We regard the forward model as a stochastic process, in which the forward propagation model $\mathbf{A}^{(0)}$ is used as a mean value in a multivariate Gaussian prior. Each of the forward fields are modeled as independent, such that the prior distribution of a single forward field is given by prior mean $\mathbf{a}_{i}^{(0)}$ (i'th column in $\mathbf{A}^{(0)}$ ) and precision $\gamma_{i}$, i.e.

$$
p\left(\mathbf{A} \mid \mathbf{A}^{(0)}, \boldsymbol{\gamma}\right)=\prod_{i=1}^{N_{d}} \mathcal{N}\left(\mathbf{a}_{i} \mid \mathbf{a}_{i}^{(0)}, \gamma_{i}^{-1} \mathbf{I}_{N_{c}}\right)
$$

where $\gamma=\left\{\gamma_{i}\right\}_{i=1}^{N_{d}}$. Assigning each of the forward fields a precision parameter allows us to differentiate between the amounts of correction of the forward fields that should be performed. This is motivated by the belief that some regions in the brain are more affected than others. Similar to the precision parameters of the current sources, the precision parameters for the forward fields are also modeled by conjugate prior distributions, i.e. $\gamma_{i} \sim \mathcal{G}\left(\gamma_{i} \mid \nu_{\gamma_{i}}, \zeta_{\gamma_{i}}\right)$. Likewise, the inverse noise variance is assumed to be Gamma distributed, $\mathcal{G}\left(\beta \mid \nu_{\beta}, \zeta_{\beta}\right)$. We note that the $\mathrm{MN}$ estimate is a special case of the SOFOMORE solution, where the prior for the forward fields are just delta functions centered at $\mathbf{a}_{i}^{(0)}$ and moreover the values of precisions for the current sources are all equal. 
Given the hierarchical structure of the SOFOMORE model with the parameters $\boldsymbol{\theta}=\{\mathbf{S}, \mathbf{A}, \alpha, \gamma, \beta\}$ the marginal posterior distribution of the current sources becomes analytically intractable, thus, approximations are needed. We apply a standard VB framework [11], in which a parameterized simpler distribution $q(\boldsymbol{\theta})$ approximates the true joint posterior $p(\boldsymbol{\theta} \mid \mathbf{M})$. In the VB framework the parameters of the $q(\boldsymbol{\theta})$ distribution are determined with a maximization of a lower bound of the marginal likelihood obtained by the Jensen's inequality,

$\mathcal{L}=\ln \int p(\mathbf{M} \mid \boldsymbol{\theta}) p(\boldsymbol{\theta}) \mathrm{d} \boldsymbol{\theta} \geq \int q(\boldsymbol{\theta}) \ln \frac{p(\mathbf{M} \mid \boldsymbol{\theta}) p(\boldsymbol{\theta})}{q(\boldsymbol{\theta})} \mathrm{d} \boldsymbol{\theta}$

We assume factorization in the parameters $\boldsymbol{\theta}=\{\mathbf{S}, \mathbf{A}$, $\alpha, \gamma, \beta\}$ of the approximate posterior $q(\boldsymbol{\theta})$. This leads to the sequential VB updates as illustrated in Fig. 1, where each of the approximated marginal posterior distributions of the parameters can be seen.

\section{EXPERIMENTS}

We demonstrate the effects of the SOFOMORE model source reconstruction for both simulated and real EEG data. We present the recovered source density estimates both with and without estimated forward fields. Besides the MN method with a fixed forward model, we also compare the SOFOMORE model with a model with a similar hierarchical structure as the SOFOMORE, however, with a fixed forward field, i.e. no A- or $\boldsymbol{\gamma}$-steps are performed. We denote this model as the ARD model, due to its ARD prior on the sources. For clarity of the results, the methods work directly on the measurements $M$, i.e. no pre-processing is performed. However, pre-processing will in general improve the performance. As validation metrics we use the mean square error (MSE), variance explained (VE), degree of focalization (DF), and area under the receiver operating curves (AUC). Definition of the validation metrics is given in Tab. 1 in section 3.1.

\subsection{Simulations}

In the simulations a small cortical area in the left occipital lope is simulated as active. The source signal consist of a half sine of duration $50 \mathrm{~ms}$ starting at $\mathrm{t}=25 \mathrm{~ms}$. The simulated sources at $\mathrm{t}=50 \mathrm{~ms}$ are shown in the SPM glass-brain representation in Fig. 2. Due to the mapping from cortex to the glass-brain representation, minor activity seems to appear at the inner part of left hemisphere. Moreover, Fig. 2 shows the time series of the simulated sources. The first 256 rows in the image are the 256 most active sources and the last 256 rows below the black horizontal line in the image are 256 randomly drawn sources with the 256 most active sources excluded. This will give an idea of the
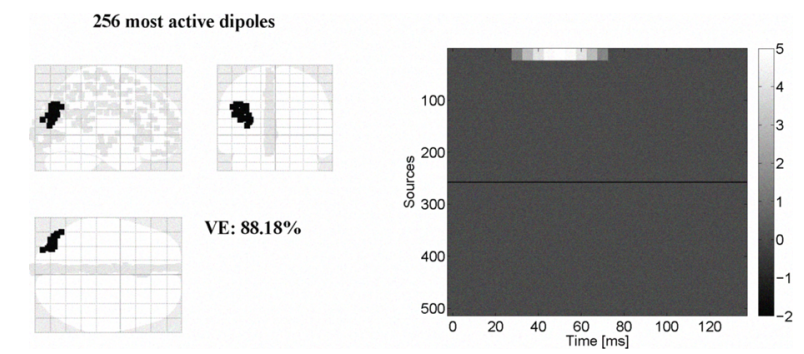

Fig. 2. Left: Simulated sources at $\mathrm{t}=50 \mathrm{~ms}$. Right: Time series of the 256 largest sources above the horizontal black line at source number 256 . Below this line are 256 randomly selected sources of the left overs to indicate the noise level.

amount of noise present in the estimated solution. Note, that since this figure shows the simulated sources, only a minor part of the most active sources are active. The corresponding simulated EEG consists of the clean EEG constructed with a 3 -spheres forward model by SPM5 ${ }^{1}$ with tissue conductivities brain:skull:scalp $=0.33: 0.0041: 0.33 \mathrm{~S} / \mathrm{m}$ (ratio 1:1/80:1) and dimension $128 \times 7204$ added with 'realistic EEG noise'. The amount of noise added to the clean EEG signal is in the order of a SNR $=10$ similar to [6], with SNR defined as the ratio between the power of the clean EEG and the noise. The noise is adopted from an evoked EEG study described in Sec. 3.2, where we have used the pre-stimulus period as noise. For source estimation three different 3-spheres head models with a lower spatial resolution than the 'true' forward model is used, and two of these are added with conductivity errors. In Fig. 3 both the location and the time series of the estimated source densities for MN, ARD, and SOFOMORE are shown. The estimated sources illustrated on the SPM glass brain correspond to the time point $\mathrm{t}=50 \mathrm{~ms}$. In this figure a forward model with 'erroneous' conductivity value for the skull $(0.0223 \mathrm{~S} / \mathrm{m})$ has been used. The MN estimate leads to a quite good localization of the main activity in the occipital lope, however, also minor activity in the right pre-motor area is reconstructed. In the image with the time series included, it is seen that the MN solution also reconstructs quite a bit of activity outside the time period $25-75 \mathrm{~ms}$ where no activity should be present. Compared to the MN solution, the source reconstruction using the ARD model is very sparse with a dominant source located correctly in left occipital region. However, the ARD model also captures minor activity in the pre-motor area. It is noted that the amplitude of the active sources are much higher than the simulated ones, since only a few account for most of the energy in the EEG signal. In contrast, the SOFOMORE model only reconstructs

\footnotetext{
${ }^{1}$ The forward model was estimated by the SPM5 academic software (http://www.fil.ion.ucl.ac.uk/spm/), based on routines from BrainStorm (http://neuroimage.usc.edu/ brainstorm/).
} 


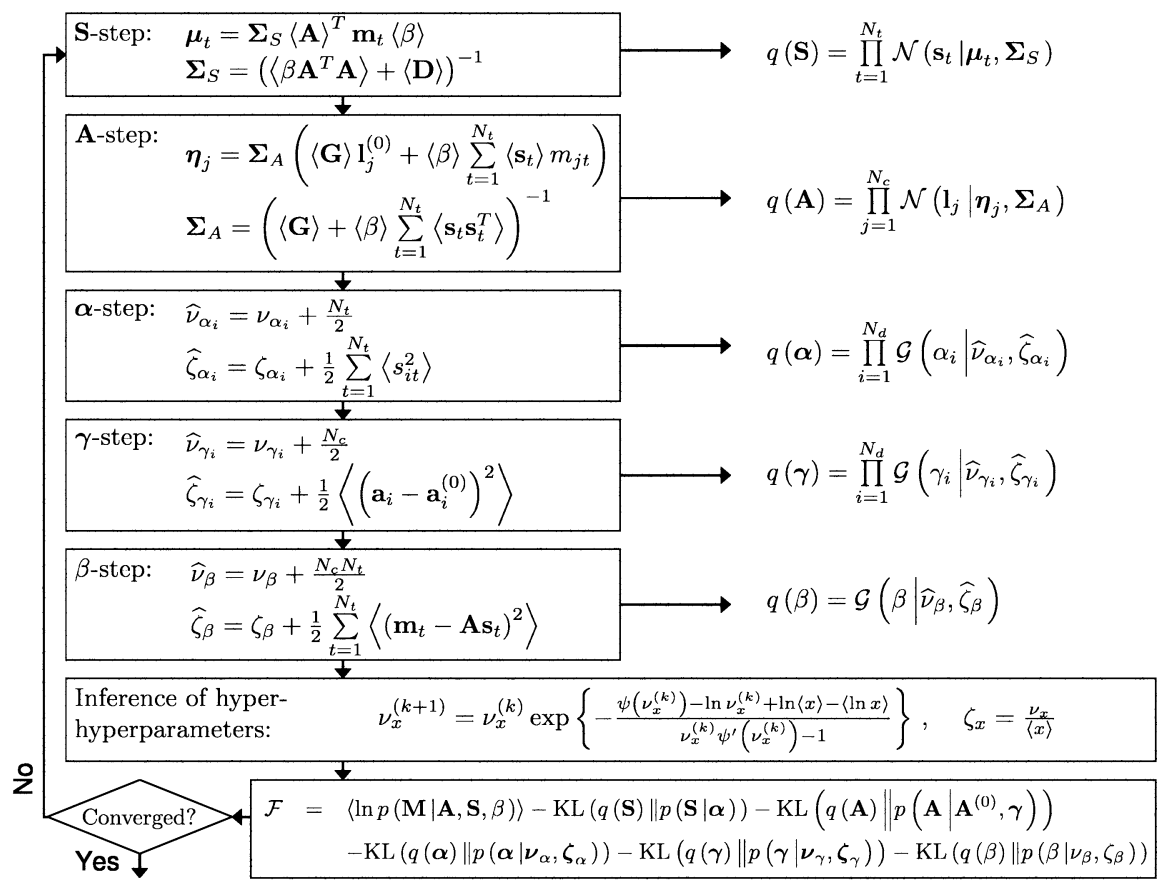

Fig. 1. Flow chart of the SOFOMORE model in a VB formulation. A fully factorized model in $\boldsymbol{\theta}=\{\mathbf{S}, \mathbf{A}, \boldsymbol{\alpha}, \gamma, \beta\}$ is applied, where each of the VB updates can be seen with their corresponding approximate posterior distribution to the right. The log evidence of the model is denoted $\mathcal{F}$, which includes Kullback-Leibler (KL) divergences of the approximate posterior distributions and their prior distributions. Following notations are used $\langle\cdot\rangle$ denoting the expectation, $\mathbf{l}_{j}^{T}$ the $\mathbf{j}^{\prime}$ th row in $\mathbf{A}$, and $\mathbf{G}$ a diagonal matrix with $\boldsymbol{\lambda}$ in the diagonal. Furthermore, $\psi(\cdot)$ is the digamma function and its derivative is $\psi^{\prime}(\cdot)$. Hyper-hyperparameters of the form $\nu_{x}$ and $\zeta_{x}$ are updated using Newton-Raphson.

the activity in the left occipital and minimal activity outside the time period of the simulated source is reconstructed. It is seen that regarding the forward fields as stochastic processes leads to an improvement relative to the source estimates obtained with fixed forward model in the ARD model.

In Fig. 4 similar source estimates with a 'correct' choice of conductivities are shown. A better estimate of the source amplitudes is shown compared to the simulated sources except for the ARD method due to its sparse nature. Indeed, an improvement of the ARD source estimates is also obtained by taking the uncertainty of the forward fields into account here, even with the 'correct' conductivity values given. This is further validated by the accuracy metrics described in Tab. 1. Table 2 shows the results for all three forward models used for source reconstruction. Note that illustrations as Fig. 3 and 4 for the third forward model with conductivity errors included (brain:skull:scalp=0.33:0.0013:0.33 $/ \mathrm{m}$ ) have been left out, since the results are quite similar to the ones in Fig. 3 and 4 with reconstruction of minor misleading activity in the pre-motor area for the MN and ARD methods. The differences can be seen from the validation metrics.
Table 1. Validation metrics.

\begin{tabular}{l}
\hline Definition \\
\hline $\mathrm{MSE}=\sum_{t=1}^{N_{t}}\left\|\hat{\mathbf{s}}_{t}-\mathbf{s}_{t}\right\|^{2} / \sum_{t=1}^{N_{t}}\left\|\mathbf{s}_{t}\right\|^{2}$ \\
$\mathrm{VE}=\sum_{j=1}^{N_{c}} \operatorname{var}\left(\mathbf{M}_{j} .-\mathbf{a}_{j}^{T} \mathbf{S}\right) / \sum_{j=1}^{N_{c}} \operatorname{var}\left(\mathbf{M}_{j}\right)$ \\
$\mathrm{DF}=\sum_{i \in \Theta}\left\|\hat{\mathbf{S}}_{i .}-\mathbf{S}_{i} \cdot\right\|^{2} / \sum_{i \in \Theta}\left\|\mathbf{S}_{i} \cdot\right\|^{2}$ \\
$\mathrm{AUC}:$ Corresponding to $p\left(\hat{\mathrm{E}}\left(i^{\prime}\right)>\hat{\mathrm{E}}(i)\right)$, with $i^{\prime}$ deno- \\
ting the index of an active source and $i$ a inactive source \\
and $\hat{\mathrm{E}}(i)=\left\|\hat{\mathbf{S}}_{i} \cdot\right\|^{2} / \max \left(\|\hat{\mathbf{S}}\|^{2}\right)$ \\
\hline \hline
\end{tabular}

\subsection{Real EEG data}

In this section real EEG data is used, which is from a multimodal study on face perception, where faces and scrambled faces were presented for $600 \mathrm{~ms}$ every $3600 \mathrm{~ms}$ to a subject. A detailed description of the experiment is given in [15] and http://www.fil.ion.ucl.ac.uk/spm (where the data is available for download). In this contribution we reconstruct the average event related potential (ERP) of trials 
Table 2. Results of validation metrics for MN, ARD, and SOFOMORE on three forward models with different conductivity ratios. SOFOMORE performs best in the metrics MSE, VE and AUC. Optimal value for VE is $\mathbf{8 8 . 2 \%}$. Note that MSE measures the ability to reconstruct the true active sources while keeping estimates of the true inactive sources small. SOFOMORE's ability to suppress the noise results in the best MSE. In contrast, DF measures only the relative squared error of the source estimates for the simulated region. Since the amplitudes of the MN estimates are higher than SOFOMORE's but still smaller than the true, MN perform best in this measure.

\begin{tabular}{l|ccc|ccc|ccc}
\hline \hline & \multicolumn{3}{|c|}{ MN } & \multicolumn{3}{c|}{ ARD } & \multicolumn{3}{c}{ SOFOMORE } \\
& $1: 1 / 15: 1$ & $1: 1 / 80: 1$ & $1: 1 / 250: 1$ & $1: 1 / 15: 1$ & $1: 1 / 80: 1$ & $1: 1 / 250: 1$ & $1: 1 / 15: 1$ & $1: 1 / 80: 1$ & $1: 1 / 250: 1$ \\
\hline MSE & 0.85 & 1.08 & 5.05 & 3.92 & 20.21 & 119.86 & 0.91 & 0.76 & $\mathbf{0 . 7 4}$ \\
VE & $99.0 \%$ & $98.8 \%$ & $98.8 \%$ & $99.5 \%$ & $99.1 \%$ & $98.7 \%$ & $95.5 \%$ & $\mathbf{9 1 . 6 \%}$ & $81.5 \%$ \\
DF & 0.76 & 0.42 & $\mathbf{0 . 3 0}$ & 2.11 & 8.03 & 78.44 & 0.88 & 0.69 & 0.56 \\
AUC & 0.93 & 0.97 & 0.98 & 0.89 & 0.93 & 0.87 & 0.92 & $\mathbf{0 . 9 9}$ & $\mathbf{0 . 9 9}$ \\
\hline \hline
\end{tabular}
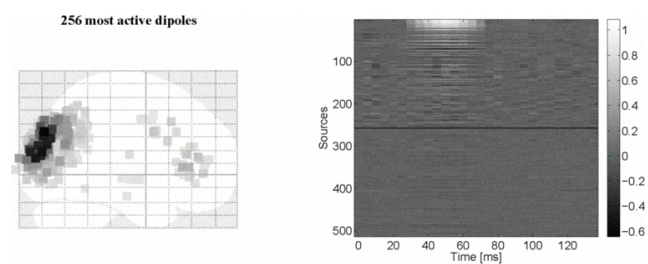

(a) $\mathrm{MN}$
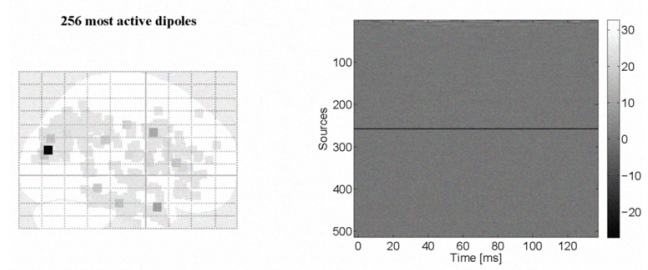

(b) ARD
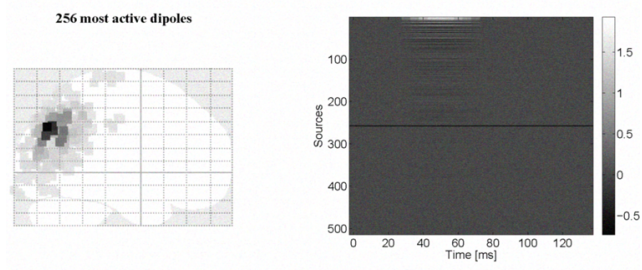

(c) SOFOMORE

Fig. 3. Source estimates when forward model with 'erroneous' conductivity values (ratios $1: 1 / 15: 1$ ) are used. Left: Activity at $\mathrm{t}=50 \mathrm{~ms}$. Right: Time series as indicated in Fig. 2 .

involving real faces as stimuli. The estimated source densities at $t=170 \mathrm{~ms}$ for MN, ARD, and SOFOMORE are illustrated in Fig. 5. Quite different results are obtained, with the three source localization methods. The MN leads to activity in a larger area compared to the ARD and SOFOMORE, with most of its activity located in the right occipital region, the right frontal region and right fusiform gyrus. The ARD results in scattered activity with two prominent voxel in the left and right temporal lope, respectively, and less prominent voxels in the visual cortex. In contrast, forward modeling in SOFOMORE leads to improved localization of activity in the visual cortex compared to the ARD with a
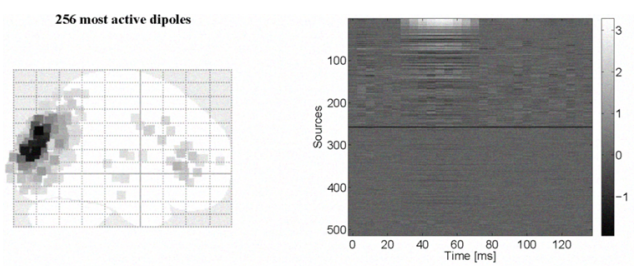

(a) $\mathrm{MN}$
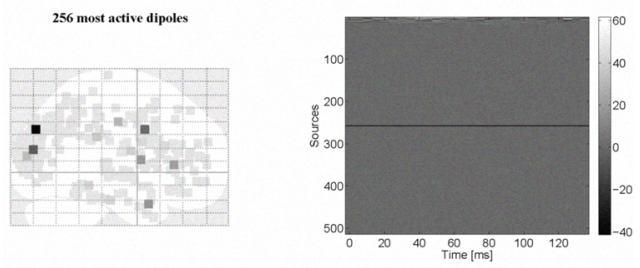

(b) ARD
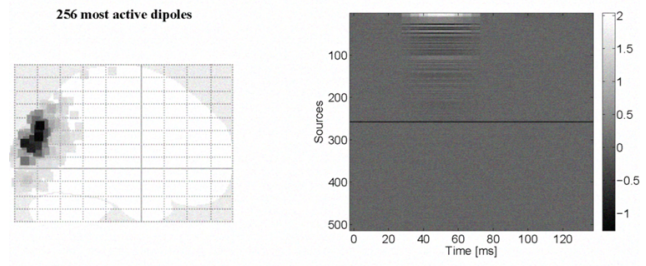

(c) SOFOMORE

Fig. 4. Similar to Fig. 3 however with 'correct' conductivity values (ratios 1:1/80:1).

fixed forward model. The source estimate in SOFOMORE is quite similar to the $\mathrm{MN}$ solution, however with a dominant region in the left visual cortex and basically no frontal activity. The SOFOMORE also leads to weak activity in both the left and right fusiform gyrus, which is known to be connected with face processing. This is well-aligned with results reported in [10].

\section{CONCLUSION}

We presented the first results of a hierarchical Bayesian framework for simultaneous source and forward model reconstruction, with no explicit physical assumptions about the 


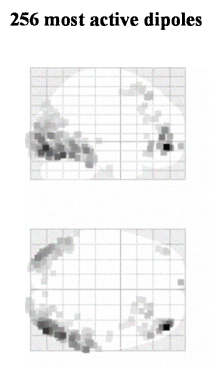

(a) $\mathrm{MN}$

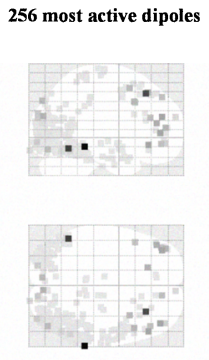

(b) ARD

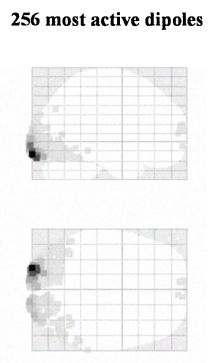

(c) SOFOMORE
Fig. 5. Estimated activity $t=170 \mathrm{~ms}$ after stimulus. Tissue conductivities brain:skull:scalp $=0.33: 0.0041: 0.33 \mathrm{~S} / \mathrm{m}$ are used.

source and forward model error. Both simulations and results from real EEG data illustrated an improvement of a similar model where the forward model is assumed fixed. Moreover, a comparison with the minimum norm method was also used to illustrate the applicability of the extended hierarchical model. Simulation results showed that the SOFOMORE model was able to reduce large distance errors. A serious concern of extending source localization methods to include forward model reconstruction is overfitting. However, the choice of an ARD prior on the forward fields allows corrections to mainly be performed where it is required to fit the signal while keeping the model simple.

\section{REFERENCES}

[1] S. Baillet, J.C. Mosher, and R.M. Leahy, "Electromagnetic brain mapping," IEEE Signal Processing Magazine, vol. 18, pp. 14-30, 2001.

[2] J. C. Mosher, R. M. Leahy, and P. S. Lewis, "EEG and MEG: forward solutions for inverse methods," IEEE Transactions on Biomedical Engineering, vol. 46, no. 3, pp. 245-259, 1999.

[3] C.H. Wolters, A. Anwander, X. Tricoche, D. Weinstein, M.A. Koch, and R.S. MacLeod, "Influence of tissue conductivity anisotropy on EEG/MEG field and return current computation in a realistic head model: A simulation and visualization study using highresolution finite element modeling," Neuroimage, vol. 30, no. 3, pp. 813-826, 2006.

[4] S. Baillet and L. Garnero, "A bayesian approach to introducing anatomo-functional priors in the EEG/MEG inverse problem," IEEE Transactions on Biomedical Engineering, vol. 44, no. 5, pp. 374-385, 1997.

[5] C. Phillips, M.D. Rugg, and K.J. Friston, "Anatomically Informed Basis Functions for EEG Source Localisation: Combining Functional and Anatomical
Constraints," Neurolmage, vol. 16, no. 3, pp. 678695, 2002.

[6] M.-A. Sato, T. Yoshioka, S. Kajihara, K. Toyama, N. Goda, K. Doya, and M. Kawato, "Hierarchical bayesian estimation for MEG inverse problem.," Neuroimage, vol. 23, no. 3, pp. 806-26, November 2004.

[7] N.J. Trujillo-Barreto, E. Aubert-Vazquez, and W.D. Penny, "Bayesian M/EEG source reconstruction with spatio-temporal priors," NeuroImage, vol. 39, pp. 318-335, 2008.

[8] J. C. Mosher, P. S. Lewis, and R. M. Leahy, "Multiple dipole modeling and localization from spatio-temporal MEG data," IEEE Transactions on Biomedical Engineering, vol. 39, no. 6, pp. 541-557, 1992.

[9] M.S. Hämäläinen and R.J. Ilmoniemi, "Interpreting magnetic fields of the brain: minimum norm estimates," Med. Biol. Eng. Comput., vol. 32, pp. 35-42, 1994.

[10] K. Friston, L. Harrison, J. Daunizeau, S. Kiebel, C. Phillips, N. Trujillo-Barreto, R. Henson, G. Flandin, and J. Mattout, "Multiple sparse priors for the M/EEG inverse problem," NeuroImage, vol. 39, pp. 1104-1120, 2008.

[11] C.M. Bishop, Pattern Recognition and Machine Learning, Springer, NY 10013 (USA), 2006.

[12] S.M. Plis, J.S. George, S.C. Jun, D.M. Ranken, P.L. Volegov, and D.M. Schmidt, "Probabilistic forward model for electroencephalography source analysis," Physics in Medicine and Biology, vol. 52, no. 17, pp. 5309-5328, 2007.

[13] S. Lew, C. Wolters, A. Anwander, S. Makeig, and R.S. MacLeod, "Low resolution conductivity estimation to improve source localization," in New Frontiers in Biomagnetism. Proc. of the 15th Int. Conf. on Biomag., 2007, vol. 1300 of Int. Congress Series, pp. 149-152.

[14] C. Stahlhut, M. Mørup, O. Winther, and L.K. Hansen, "SOFOMORE: Combined EEG source and forward model reconstruction," in 6th IEEE Int. Symp. on Biomed. Imaging (ISBI): From Nano to Macro, 2009, pp. $450-453$.

[15] R.N.A. Henson, Y. Goshen-Gottstein, T. Ganel, L.J. Otten, A. Quayle, and M.D. Rugg, "Electrophysiological and hemodynamic correlates of face perception, recognition and priming," Cerebral Cortex, vol. 13, pp. 793-805, 2003. 